\title{
Abdominal Fluid
}

National Cancer Institute

\section{Source}

National Cancer Institute. Abdominal Fluid. NCI Thesaurus. Code C77611.

The fluid that is abnormally collected in the abdominal cavity as a result of vascular pressure, liver disorder, or malignancy. 